\title{
INDEX AND GLOSSARY
}

Chinese names in transcription are followed by Chinese characters, unless they occur with reference to publications in alphabetic languages only (e.g. Dai Sijie, Kong Shuyu). Life years are included for all wellknown avant-garde poets mentioned in this study (see p xiv).

Terms and expressions are listed in Chinese as well as English if they are originally Chinese (e.g. wen yi zai dao 文以载道 'literature to convey the Way') or have a specifically Chinese usage (e.g. xianfeng 先 锋 'avant-garde').

Texts listed include unofficial poetry journals (e.g. Today / Fintian《今 天》, Poetry Reference / Shi cankao 《诗参考》) and several serial book publications (e.g. Epoch Poetry Series / Niandai shicong《年代诗从》), in recognition of their importance for the avant-garde; and a few canonical texts from antiquity, whose significance is undiminished today (e.g. Songs of the South / Chuci《楚辞》).

A Cheng 阿城: 379

A Fei 阿斐 (1980): 335

A Jian 阿坚 (1955): 406

Abraham, Nicolas: 298

Abrams, Meyer Howard: 347, 360

abusive criticism (骂、骂人): 425, 448

Academicized Writing (泛学院化写 作): 334

Adorno, Theodor: 416

Ah Q阿Q: 404

Ai Qing 艾青: 4, 102, 106

Akhmatova, Anna: 6, 416

Alternative Poetry (另类诗歌): 20

Alvarez, Alfred: 91, 94, 113

Analects (论语): 261

anti-intellectualism: 193, 318, 321, 353, 382, 391, 411, 442

Apollinaire, Guillaume: 403

Arbus, Diane: 315

Ash, Adrienne: 146

Ashbery, John: 220, 404-405

Attridge, Derek: 33, 284
Auden, Wystan Hugh: 403

author, historical person of: 50-51, 93-95, 123-124, 149, 161, 164, 192, $196,321,443-446$

authorial intent: 51, 93, 190, 196, 345-346, 376

avant-garde (先锋): chapter One (esp 5-12) et passim

Aviram, Amittai: 59, 285, 288, 298-304

Ba ling hou shixuan 《八○后诗选》 $\rightarrow$ Selected Post-80 Poetry

Ba ling hou yi dai 八O后一代 $\rightarrow$ Post-80 Generation

bad behavior: 305, 334, 339-341

Bai Hua 柏桦 (1956): 83, 103, 402

Bai Ye 白夜 (= Duoduo): 7n8

Bailey, Alison: 113

Barmé, Geremie: 8, 148, 319

Barnstone, Tony: 287

Baudelaire, Charles: 6, 132, 226, 229-230, 243, 375 
Beauty Writers (美女作家) $\rightarrow$ Glamlit Writers

Bei Dao 北岛 (1949): xiv-xv, 7, 15-16, $18,26,31,34,39,42,52,55,66$, 68n7-69, 71, 83n26, 89, 91, 93n1, 95, 106, 239, 247, 254-255, 341, 378-379, 406, 408; chapter Four; $\rightarrow$ Zhao Zhenkai

Beiling 贝岭 (1959): xiv, 139, 141; $\rightarrow$ Huang Beiling

Bell, Robert: 146, 179

benbanshu 本版书 $\rightarrow$ original editions

Bertens, Hans: 191

Bevan, David: 143, 160

Bian Zhilin 市之琳: 4

Bing Xin 冰心: 4

Birch, Cyril: 287

Blue Star Poetry Treasure House (蓝星诗 库): 247, 249, 281

book agents (书商) $\rightarrow$ book brokers

book brokers (书商): 12

Book of Songs (诗经): 1, 185, 424

Borges, Jorge Luis: 189, 403, 416

Bourdieu, Pierre: 11, 22, 34, 36, 47, 49, 57, 144, 191, 346, 392, 448

Boym, Svetlana: 160, 178

Bradbury, Steve: 60

Brems, Hugo: 51

Breton, André: 403

Brodsky, Joseph: 160, 178, 214, 385, 403, 406

Bronfen, Elisabeth: 93

Brouwers, Jeroen: 91

Brown, Edward: 161

Bruno, Cosima: 251

Burroughs, William: 467-468

Buruma, Ian: 160, 178

Bush, George Herbert Walker: 149

Bush, George Walker: 148

Byron, George Gordon: xiii, 107, 109, 121,412

Cai Kelin 蔡克霖: 68-69

Cai Yi 蔡毅: 33, 250-251

Campus Poetry (校园诗歌): 65-66

Can Xue 残雪: 247

Cardinal, Roger: 220

Cayley, John: 154

Cao Pi 曹丕: 448

Cao Shuying 曹疏影 (1979): 461

Celan, Paul: 108 censorship: 5, 8, 14, 22, 143, 147-150, 153-159, 162, 168, 175-176, 287, 307, 330, 336, 417

Chang Li 常立: 101, 113, 379

Chang Yao 昌耀: 113

changduanju 长短句 $\rightarrow$ long-short line

Che Qianzi 车前子 (1963): iv, 20, 48, 461, 473

Cheesman, Tom: 143

Chen Chao 陈超: xiv, 33, 120-121, 129-130, 168, 399, 423, 425, 432

Chen Dongdong 陈东东 (1961): 17, 83, 100-103, 135, 169, 178, 189-190, 230, 402-403, 406, 443-444

Chen Duxiu 陈独秀: 3

Chen Jingrong 陈敬容: 4

Chen Jun 陈均: 420, 423, 440

Chen Qufei 陈去非: 250-251

Chen Ran 陈染: 247

Chen Zhongyi 陈仲义: 77, 251-252, 333

Cheng Guangwei 程光炜: 102, 105, $124,161,168,281$; passim in chapter Twelve (esp 400-405, 434-435)

Cheng Weidong 程蔚东: 71

Chi Li 池莉: 247

Chinese Writers' Association (中国作家 协会): 4, 11, 260, 272-273, 390, 418

Chineseness: 54-59, 136, 143, 386-389; passim in chapter Twelve (esp 443-444)

Choushui 仇水 (= Shen Haobo): 405

Chow, Rey: 54-55, 59

Chuci 《楚辞》 $\rightarrow$ Songs of the South

Chung, Hilary: 161, 178

chunshi 纯诗 $\rightarrow$ pure poetry

ci 词 $\rightarrow$ lyric

citation repository: $\mathrm{xv}, 475$

close reading (definition): 59

Coleman, Steve: 244

Coleridge, Samuel: 228

Colloquial Poetry (口语诗) and colloquialization: $16,18,52,78,80$, 88, 135, 189, 252, 278, 310, 362, $365,373,439,445$

commercialization: 14-15, 32-33, 36, 46-47, 49, 111, 119, 161-162, 187, 191-196, 203, 209, 214, 221, 321-322, 337, 352, 362, 382-384, 415, 433, 438-439, 442, 447, 450

Confucius 孔子: 359 
“connecting” (接轨) with the West: 57 , 386-387, 416, 428, 433, 444

content: 288 (definition); $\rightarrow$ form and content....

content bias: 284-287, 302-303

Contemporary Chinese Experimental Poetry (中国当代实验诗歌): 95

context $\rightarrow$ text, context and metatext

Cooper, Burns: 296

Coquetry Poetry Fournal (撒娇诗刊): 38

Coquetry School (撒娇派): 17, 42

Crespi, John: 12, 36, 251, 283, 473

crisis discourse: 32-33, 119, 305, 335

Cui Jian 崔建: 107n21

Cui Weiping 崔卫平: 102, 118, 194, 197, 214

Culler, Jonathan: 33, 107, 293

cult of poetry: 18, 37, 46, 52, 103-104, 108, 112-113, 115, 190, 197, 204, 349, 365-366, 370, 372, 382, 394

cultural economy (文化经济): 32, 36, $48-49,473$

Curtis, Ian: 94

DACHS: xv, 472, 475

Dai Sijie: 162n46

Dai Wangshu 戴望舒: 4, 403

Dark Skin Poetry Series (黑皮诗丛): 247

Dawei 大卫 (= Yu Jian): 262

Daxu 〈大序〉 $\rightarrow$ "Great Preface"

Day, Michael: 21-22n29, 53, 60, 103, 113, 338, 341

De Haan, Martin: 306

De Meyer, Jan: 251, 385

De Roder, Jan: 299, 347

DeFrancis, John: 193

demystification: $37,46,71,80,168$, 214, 305, 338-339, 341, 365, 438, 445

Deng Xiaoping 邓小平: 14, 149, 230

Denton, Kirk: 59, 107

Descartes, René: 461

di er qudao 第二渠道 (= er qudao二渠道) $\rightarrow$ second channel

Di san dai 第三代 $\rightarrow$ Third Generation

Di shige yundong 低诗歌运动 $\rightarrow$ Low Poetry Movement

Di si dai 第四代 $\rightarrow$ Fourth Generation

dialect: 267, 373-374, 376, 387-388, 407-408, 410

Digital Archive for Chinese Studies $\rightarrow$ DACHS
Dilthey, Wilhelm: 417

Ding Dang 丁当 (1962): 72, 261

Ding Mang 丁芒: 431

dixia 地下 $\rightarrow$ underground

Don Quixote: 404

$\mathrm{Du} F \mathrm{Fu}$ 杜甫: 1

Duanlie 断裂 $\rightarrow$ Rupture

Duchamp, Marcel: 240, 323

Duoduo 多多 (1951): xiv, 7, 18, 119, 137-139, 146, 150, 157, 170-171, 406; $\rightarrow$ Bai Ye

Duoyu 朵渔 (1973): 305, 335, 393

Eagleton, Terry: 229

Earthly aesthetic: 10, 18, 20, 23-27

(definition), 29-30, 37-38, 45,

52-53, 73, 125, 168, 187, 190, 193, 221, 223, 251-252, 267, 281, 305, 314, 318, 331, 333-334, 338-339, 341-342, 346, 363, 365, 372, 382, 392, 394, 396-397, 399, 427, 438, 445,447

Easthope, Anthony: 50, 53, 223, 459

Edmond, Jacob: 153, 161, 178

Edwards, Robert: 141

Elevated aesthetic: 10, 18, 20, 23-27 (definition), 30, 37, 45, 47, 52-53, 73, 79, 125, 135, 187, 189-190, 193, 197, 218, 221, 223, 251, 281, 305, 334, 338-339, 341-342, 346, 363, 365, 370, 372, 394, 396-397, 399, 445,447

Eliot, Thomas Stearns: 286, 299, 403-405

Enlightenment (启蒙): 15, 444

Eoyang, Eugene: 160

Epoch Poetry Series (年代诗丛): 63, 247, 438, 449

er qudao 二渠道 (= di er qudao 第二渠 道) $\rightarrow$ second channel

establishment: 6 ; $\rightarrow$ orthodox poetics

exile and exile literature: $18,52,54,57$, 59, 287, 385-386, 406, 410, 414, 421; chapter Four (definitions: 141-143, 146-147, 158-161, 177-179)

experimental (实验): 10

explicit poetics: 10, 13, 37-38, 51-52, 65, 71-73, 95, 108, 122, 124n47, $152,164,180,213,250-251,263-$ 265, 267, 283, 303, 315, 315-318, 331, 338, 409, 416, 439, 442-443; 
passim in chapters Ten (esp 345-347, definition: 345) and Eleven

explorative (探索): 10

Fan xueyuanhua xiezuo 泛学院化写作 $\rightarrow$ Academicized Writing

Fang Lizhi 方励之: 149

Fang Xiang 方向: 104

fangyan 方言 $\rightarrow$ dialect

Faulkner, William: 416

fei guanfang 非官方 $\rightarrow$ unofficial

Fei Jia 费嘉: 260, 262

Fei Sha 飞沙 (= Yang Lian): 7n8, $147 \mathrm{n} 15$

Feifei 《非非》 $\rightarrow$ Not-Not....

feminist critical discourse $\rightarrow$ Women's Poetry....

Feng Zhi 冯至: 4

Ferry, Megan: 321-322

Findeisen, Raoul: 101

Finkel, Donald: 287

First Line (一行): 141

fm3: 23, 462-463, 465, 472

foreign literatures, modern Chinese poetry's relation to: $3,6,15,55-59$, 123, 136, 152-153, 155-156, 162, 266, 362, 375, 379, 385-390; passim in chapter Twelve

form: 299 (definition); $\rightarrow$ form and content....

form and content, synergy of: 52, 59, 125, 200-201, 215-221, 274-280, $325,465-467$; passim in chapters Six and Eight

Forrest-Thomson, Veronica: 284, 286287, 299

Foucault, Michel: 416

Fourth Generation (第四代): 17

Freud, Sigmund: 298

Frost, Robert: 387

Frye, Northrop: 225, 293

$f u$ 赋 $\rightarrow$ poetic exposition

Fu Mahuo 符马活 (1970): 330-331

Futurism: 342

Gao Bo 高波: 102-103, 105, 122, 123， 251

Gao Xingjian 高行健: 148, 161-162

Gaoyuan shïi 《高原诗辑》 $\rightarrow$ Highland Poetry Compilation

Garbage School (垃圾派) $\rightarrow$ Trash School
Ge Mai 戈麦 (1967-1991): 98, 100-101, 103n14, 111, 196, 247,381

Geng Zhanchun 耿占春 (1957): 412， 440

Gerbrandy, Piet: 125

Geren xiezuo 个人写作 $\rightarrow$ Individual Writing

Gerenhua xiezuo 个人化写作 $\rightarrow$ Individualized Writing

Gillespie, Marie: 143

Ginsberg, Allen: 467-468

Glad, John: 59, 143, 146-159, 183

Glamlit Writers (美女作家): 320-322

Glass, Philip: 466

Glazier, Loss Pequeño: 22n29, 244

Goethe, Johann Wolfgang von: 98, 245

Gorky, Maksim: 468

Graham, Angus: 382n37

“Great Preface" (大序): 185

Gu Cheng 顾城 (1956-1993): 7, 26, 31, 34, 91, 93n1, 98, 100-101, 103, 112-113, 139, 147, 163, 247, 249, 287, 381, 391, 414, 438

guanfang 官方 $\rightarrow$ official

guanxi 关系 $\rightarrow$ ties of allegiance

Gui Xinghua 贵兴华: 24, 48

Guo Lusheng 郭路生 (= Shizhi, 1948): xiv, 15, 101; $\rightarrow$ Shizhi

Guo Moruo 郭沫若: 4, 106, 110, 125

Guo Xiaochuan 郭小川: 102, 130, 136, 275,408

Ha Jin: 162n46

Haft, Lloyd: 3, 287, 446

Haizi 海子 (1964-1989): xiii-xiv, 17, 31, 37, 39, 43, 52, 189-190, 196, 206-208, 210, 214, 218, 247, 249, 303, 333, 339, 365, 375, 381, 408, 414, 430, 438, 445-446, 461; chapter Three; $\rightarrow$ Zha Haisheng

Hamlet: 404-405

Han Dong 韩东 (1961): xiii, xv, 17, 45, 51-52, 102, 116-117, 120, 168, 252, 257, 259, 261, 278, 309-310, 315, 318, 338-339, 346-347, 361, 363; chapters Two and Eleven; passim in Twelve (esp 436-439)

Han Poetry (汉诗): 17

Hanne, Michael: 160

Hanshi 《汉诗》 $\rightarrow$ Han Poetry 
Havel, Václav: 416

He Jingzhi 贺敬之: 102, 130, 136, 230, 275, 408

He Qifang 何其芳: 4

He Qinglian 何清涟: 14

He Xiaozhu 何小竹 (1963): 399, 436-437, 440

He Yi 贺奕: 223, 250-251

Hei Dachun 黑大春 (1960): 22, 37, 464-465

Hei pi shicong 《黑皮诗从》 $\rightarrow$ Dark Skin Poetry Series

Heine, Heinrich: 207

Heraclitus: 219

Hettinga, Tsjêbbe: 358

high culture fever (文化热): 13, 35, 162, 187

Highland Poetry Compilation (高原诗辑): 262

Hinton, David: 145

Hockx, Michel: 21, 123, 448

Hoffmann, Peter: 287

Hölderlin, Friedrich: 108

Holton, Brian: 60

Homer: 152, 358

Hong Kong poetry: 53

Hong Zicheng 洪子诚: 32, 83, 101-102, 281, 400, 406

Hooligan Literature (流讯文学、㾙子 文学): 321, 334, 339

Hou kouyu 后口语 $\rightarrow$ Post-Colloquial

Hou Ma 侯马 (1967): 318, 406, 414, 425, 427

Hou menglongshi 后朦胧诗 $\rightarrow$ PostObscure Poetry

Hou xin shichao 后新诗潮 $\rightarrow$ Post-NewTide Poetry

Houssaye, Arsène: 226, 243

Hu Dong 胡冬 (1962): 139

Hu Kuan 胡宽 (1952-1995): 438

Hu Shi 胡适: 3, 63, 107

Hu Tingwu 胡廷武: 251

Hu Xudong 胡续冬 (1974): 83, 334, 473

Hu Yan 胡彦: 251

Hu Yaobang 胡耀邦: 156

Huang Beiling 黄贝岭 (= Beiling): xiv

Huang Canran 黄灿然 (1963): 178

Huang Fan: 65, 71

Huang Heqing 黄河清: 163

Huang Liang 黄粱: 249, 251
Huang Lihai 黄礼孩 (1971): 331

Huang Nubo 黄怒波 (= Luo Ying): 32

Huang Rui 黄锐: 16

Huang Xiang 黄翔 (1943): xiv, 15, 139, 444

Huang Yibing 黄亦兵 (= Mai Mang): 461

Huang Yunte: 55

Huang Zunxian 黄遵宪: 107, 403

Hung, Eva: 190

Huot, Claire: 251

Hussein, Saddam $\rightarrow$ Saddam Hussein

Image Puzzle (象国): 444

indeterminacy: 52, 59, 239, 283, 359; chapter Five (definition: 197)

Individual Writing (个人写作): 19, 102, 187, 362, 434

Individualized Writing (个人化写作): 19

Intellectual Writing (知识分子写作): 17-18, 20, 190, 331-332, 338, 349, 365, 371, 375, 391-393; chapter Twelve

Intellectual Youths (知识青年): 5-6, 15

Internet: 21-23, 47, 54, 330, 332-333, $362,400,474$

Inwood, Heather: 12, 21-22, 338

Jakobson, Roman: 240, 298

Jameson, Fredric: 77

Janssen, Ronald: 23, 172, 180

Jenner, William: 55, 155

Jiang Hao 蒋浩 (1972): 334, 423

Jiang He 江河 (1949): 34, 135, 139, 341

Jiang Tao 姜涛 (1970): 334, 440

Jiangjiang 江江: 172

jiegui 接轨 $\rightarrow$ “connecting”....

Jin Xiaofeng 金小风: 393

Jing Wen 京文 (= Zhang Qinghua): 421

Jing Yi 静矣: 420-421

Jingbute 京不特 (1965): 17, 139

jingshen wuran 精神污染 $\rightarrow$ spiritual pollution

fintian 《今天》 $\rightarrow$ Today

Fiushi niandai 《九十年代》 $\rightarrow$ Nineties

Fiushi niandai shige 九十年代诗歌 $\rightarrow$

Poetry of the Nineties

Fireuxing de xiezuo 及物性的写作 $\rightarrow$

Transitive Writing

Jones, Andrew: 55 
June Fourth (六 ・四): 8, 14, 18-19, 52, 72, 98, 103-104, 113, 187, 196, 198, 385-386, 411; passim in chapter Four

Kafka, Franz: 6, 403, 406, 416

Kaiyu 开愚 $\rightarrow$ Xiao Kaiyu

Kao, Anne $\rightarrow$ Liu Li'an

Kerouac, Jack: 6

Knight, Deirdre Sabina: 321-322

Kong Shuyu: 9n9, 321-322

Kongzi 孔子 $\rightarrow$ Confucius

Kouyu shi 口语诗 $\rightarrow$ Colloquial Poetry....

Krämer, Oliver: 147-148

Kristeva, Julia: 298

Krol, Gerrit: 229, 241-244, 294

Kubin, Wolfgang: 110, 161, 172

Kui $《$ 葵》 $\rightarrow$ Sunflower

Lacan, Jacques: 298

Lacoue-Labarthe, Philippe: 298

Lajipai 垃圾派 $\rightarrow$ Trash School

Lan Dizhi 蓝棣之: 193, 197, 314, 353

Lan xing shiku 《蓝星诗库》 $\rightarrow$ Blue Star Poetry Treasure House

Lao Mu 老木: 96, 368, 444

Lao She 老舍: 5

Larson, Wendy: 63-65, 71, 77, 321-322

Lee, Gregory: 23, 55, 57

Lee, Leo Ou-fan: 25, 161, 172-173, 443

Lee, Mabel: 148, 162, 164n49, 166

Lei Feng 雷锋: 130, 230

Leng Shuang 冷霜 (1973): 83

Lenin, Vladimir Ilyich: 466

Lennon, John: 468

Lermontov, Mikhail: 68, 412

Li Bai 李白: 1, 286, 299, 354, 413, 426, 468,471

Li Bo 李勃: 260, 262

Li Chao 李超: 101-102, 110

Li Dian: 158-159n40, 161, 172-173, 379,450

Li Duoduo 栗多多: xiv

Li Fukang: 190

Li Hongqi 李红旗 (1976): 40, 335

LiJi 李季: 230, 239

LiJinfa 李金发: 4, 403

$\mathrm{Li} \mathrm{Li}$ 李笠: 156n36, 162n46

$\mathrm{Li} \mathrm{Ou}-\mathrm{fan}$ 李欧凡 $\rightarrow$ Lee, Leo Ou-fan

Li Qingzhao 李清照: 1

Li Shangyin 李商隐: 1, 403
Li Shaojun 李少君: 281

Li Shijiang 李师江 (1974): 335

Li Xia: 112

Li Yawei 李亚伟 (1963): 17, 259, 339-340

Li Yu 李显: 2, 403

Li Zhen 李震: 251

Liang Qichao 梁启超: 342

Liang Xiaobin 梁小斌 (1955): 34

Liang Xiaoming 梁晓明 (1963): 461

Liao Yiwu 廖亦武 (1958): 38, 230

Liaoyuan 燎原: 97n7, 102, 105, 114-115, 118, 120, 124

Lin Mang 林莽 (1949): 421, 425

Lin Xingqian 林幸谦: 161, 164, 172, 175

Lin Yiliang 林以亮: 226, 246

Ling dian ditie shicong 《零点地铁诗 从ᄊ》 $\rightarrow$ Midnight Subway Poetry Series

Lingenfelter, Andrea: 60, 219

Linglei shige 另类诗歌 $\rightarrow$ Alternative Poetry

Link, Perry: 55, 151

literary men disparage one another... (文人相轻....): 448

Literary Revolution (文学革命): 3, 63

literature to convey the Way (文以载 道): 2, 30, 49, 253, 352, 448

Liu Bannong 刘半农: 229

Liu Binyan 刘宾雁: 162-163

Liu Fuchun 刘福春: 440

Liu, James: 360

Liu Jun 刘军 (= Xi Chuan): 210

Liu Kexiang 刘克襄: 229

Liu Li'an 刘丽安: 413

Liu Ligan 刘立杆 (1967): 368, 373, 379, 383

Liu Na 刘纳: 192, 194, 203

Liu Shijie 刘士杰: 251

Liu Shuyuan 刘树元: 68n7, 70

Liu si 六・四 $\rightarrow$ June Fourth

Liu Xiangdong 刘向东: 420

Liumang wenxue 流讯文学 $\rightarrow$ Hooligan Literature

long-short line (长短句): chapter Seven (esp 274-280)

Longfellow, Henry Wadsworth: 387

Lovell, Julia: 46, 55, 65

Low Poetry Movement (低诗歌运动): 20,338, 341 
Lowell, Robert: 403-405

Lower Body, Lower Body (下半身): 20, 27, 29, 31, 38, 47, 52, 267, 405, 449, 468; chapter Nine

Lü De’an 吕德安 (1960): 72, 83-84, 408, 416

Lu Jie: 321

Lu Shourong 卢寿荣: 101, 113

Lu Xun 鲁迅: 11, 107, 229, 390

Lu Yang 鲁羊 (1963): 385

Lu Yimin 陆忆敏 (1962): 72, 408

Lü Zhouju 吕周聚: 9

Lunyu 《论语》 $\rightarrow$ Analects

Luo Gaolin 罗高林: 230, 245

Luo Hanchao 骆寒超: 67n4

Luo Jiasheng 罗家生: 274

Luo Qing 罗青: 225

Luo Yihe 骆一禾 (1961-1989): 39, 43, 95-124, 189, 196, 206-207, 214, 247

Luo Ying 骆英: $32 ; \rightarrow$ Huang Nubo

Luo Zhenya 罗振亚: 9, 102, 124, 281, 303, 319n 14, 334, 450

Lyne, Sandra: 321-322

lyric (词): 274

$m a$ 骂, ma ren 骂人: $\rightarrow$ abusive criticism

$\mathrm{Ma}$ Ce 马策: 332-333, 336

Ma Fei 马非 (1971): 335

Macho Men, Macho Men (芙汉): 17, 52, 193, 259, 267, 339, 445

MacLeish, Archibald: 303

Mai Mang 麦芒 (1967): 461; $\rightarrow$ Huang Yibing

male dominance of metatext: 46, 347, 366, 399

Malingshu Xiongdi 马铃薯兄弟 (1960): 391, 395

Mallarmé, Stéphane: 71, 240, 362

Mandelstam, Osip: 403

Mang Ke 芒克 (1950): 7, 16, 34, 38-39， 42, 254

Manghan 《䒭汉》 $\rightarrow$ Macho Men

Mao Dun 茅盾: 101

Mao Jian 冒键: 91, 105

Mao Style (毛文体): 5

Mao wenti 毛文体 $\rightarrow$ Mao Style

Mao Zedong 毛泽东: 2, 4, 5, 230, 369, 468

Maoist poetics $\rightarrow$ orthodox poetics

Maospeak (毛文体) $\rightarrow$ Mao Style

marginality: ix, 3, 32, 35, 46-49,
119, 159, 161, 177, 209, 213, 332,

388-390, 408, 428-429, 447

Marinetti, Filippo Tommaso: 342

May Fourth (五四): 9, 369

McDougall, Bonnie: 55, 58-59, 89n32, 154, 175, 180-181n80, 379

Meinü zuojia 美女作家 $\rightarrow$ Glamlit Writers

metatext $\rightarrow$ text, context and metatext

methodology $\rightarrow$ theory and methodology

Meng Lang 孟浪 (1961): 139

Menglongshi 朦胧诗 $\rightarrow$ Obscure Poetry

Mi Jiayan: 113

Mian Mian 棉棉: 321-322

Middle Generation (中间代): 17

Middle-Aged Writing (中年写作): 430

Midnight Subway Poetry Series (零点地铁 诗丛): 247

Mill, John Stuart: 293

Miłosz, Czeslaw: 403

Min, Anchee: 321

mind, mayhem and money: 13-14

Minjian xiezuo 民间写作 $\rightarrow$ Popular Writing

Mishima Yukio: 93

Mo Fei 莫非 (1960): 169, 406

Mo Yan 莫言: 247

Modern Han Poetry (现代汉诗): 19

Modern Poetry Materials for Internal Exchange (现代诗内部交流资料): 95

Modern Standard Chinese (普通话) $\rightarrow$ Standard Language

Momo 默默 (1964): 17, 38-39

Morewedge, Rosemarie: 178

Mou Sen 牟森: 224

Mozart, Wolfgang Amadeus: 350

Mu Qing 穆青 (1973): 461

Muguang 目光 $\rightarrow$ Vision

Nanfang shizhi 《南方诗志》 $\rightarrow$ Southern Poetry Review

Nanren 南人 (1970): 335

Narrative Poetry (叙事诗) and narrativity: 20, 52; chapter Eight (definition: 283)

nationalism: 33, 387-389, 405, 410, 412, 416, 426, 433, 435

Neruda, Pablo: 403

New Culture Movement (新文化运 动): 31 
New Literature (新文学): 9

New Period (新时代): 65

New Poetry (新诗): 3-4, 418-419, 435

New Tide Poetry (新诗潮): 421, 427429

New Youth (新青年): 3

Newborn Generation (新生代): 17, 113

Niandai shicong 《年代诗从》 $\rightarrow$ Epoch Poetry Series

Nietzsche, Friedrich: 298

Nijhoff, Martinus: 237

Nineties (九十年代): 198

Niu Han 牛汉: 71, 423

North and South, opposition of: 373 , $376,407,410,424,433,444-445$

Not-Not, Not-Not (非非): 17, 20, 26, 75, 193, 259, 338-339, 409, 438, 445

Nüxing shige 女性诗歌 $\rightarrow$ Women's Poetry....

Oates, Joyce Carol: 72

objectification: 28, 52; chapter Seven (esp 252-274)

objectivism: 77-79

Obscure Poetry (朦胧诗): 16-18, 26, 31-32, 34-35, 45, 51-52, 57, 65-76, 78-79, 88, 91, 95, 130, 135, 147, 151, 168, 189-190, 252, 254, 256, 259, $262,303,320,334,338,341,365$, $368,370,379,408,417,423,448$

obstruction (遮蔽): 425-427, 441-442, 444, 449

official (官方): 5-12; $\rightarrow$ orthodox poetics

Oosterhoff, Tonnus: 61, 287

original editions (本版书): 48

Original Writing (原创性写作): 331, 340, 468

orthodox poetics: 4-12, 16, 24, 31, 48$49,51,63,65,68,71,89,102,110$, $113,125,130,132-133,136,139$, 141, 143-144, 149, 159, 161, 177, 192, 197, 230, 245, 275, 320, 339, 341-342, 352, 361-362, 369-370, 382, 389, 402, 408-411, 413, 415, 418, 421, 425, 427-428, 430, 441

Ouyang Jianghe 欧阳江河 (1956): 83, 139, 157, 168-169, 172, 189-190, 230, 251, 298, 309, 402, 406, 408, $416,427,430,432,443-444$
Oversteegen, Jaap: 288

Ovid: 178

Owen, Stephen: 2, 55, 123, 155, 179, 185n84, 354, 359

Panfeng Poetry Conference (盘峰诗会): 309, 417-432, 439

Panfeng shihui 盘峰诗会 $\rightarrow$ Panfeng Poetry Conference

Parker, Charlie: 244

Pasternak, Boris: 153, 341, 403, 406, 414

Patton, Simon: 60, 65, 93n2, 163, 243, 251, 253, 368n7, $37 \ln 14$

peer journals (同仁刊物、同人刊物) $\rightarrow$ soulmate journals

Perelman, Bob: 35

Perloff, Marjorie: 27, 59, 189, 197, 220, 246

Pizi wenxue 㾂子文学 $\rightarrow$ Hooligan Literature

Plath, Sylvia: 108, 403

poethood: 10, 16, 18, 28, 30-50, 145146, 177-179, 187-197, 203, 214215, 305, 338-339, 343, 468; passim in chapters Three (esp 91-123), Ten (esp 348-350), Eleven (esp 366-392) and Twelve (esp 446-448)

poetic discourse (definition): 50

poetic exposition (赋): 264

poetic prose: 225

poetic rhythm: 52, 59, 199, 227;

chapter Eight (esp 285, 298-300)

poetic sentiment (诗意): 83, 87, 317318, 336, 351

poetic voice (definition): 50

poetry (definitions): 2, 61, 71-72, 82-83, 191, 253, 318, 330, 335-337, 349; passim in chapters Six (esp 225-229), Eight (esp 284-285, 298-299), Ten (esp 353-359) and Eleven (esp 366378)

Poetry and People (诗歌与人): 331, 449 poetry articulates what is on the mind intently (诗言志): 2, 228, 253, 354, 383, 396

poetry as religion: $18,37,107,109-111$, 190, 195, 303, 348-349, 354, 356359, 367-372, 394, 439, 445; $\rightarrow$ cult of poetry 
Poetry of the Nineties (九十年代诗歌): 13, 83-84, 281, 334, 401-406, 413, 419-420, 429, 434, 440

Poetry Reference (诗参考): 331, 340, 429, 431, 433, 440-441

poetry scene (诗坛, definition): 6

Poetry Text (诗文本): 38-39, 331, 449

Poetry Vagabonds (诗江湖): 331-332

poetry verbalizes emotion (诗言志) $\rightarrow$ poetry articulates....

Poets' Writing (诗人写作): 371-372, 428

Polemic, Popular-Intellectual: 11, 20, 27, 29, 37-38, 46, 52-53, 83, 107, 153, 190, 251, 281, 307, 309, 314, $318,320,331,338,345,349,353$, 365, 370-371, 382, 388, 392-393, 397, 468; chapter Twelve

Political Lyricism (政治抒情诗): 4, 24, 125, 130, 136, 145, 408

politics and literature: 2, 4, 13-15, 76, 103, 135, 287, 352, 361-362, 379-380, 385, 388, 417, 442, 447; passim in chapter Four

Popular Writing (民间写作): 20, 25， 47, 338, 365, 382, 388, 392; chapter Twelve

Post-70 Generation (七○后一代): 17, 38, 331, 334

Post-80 Generation (八○后一代): 17-18, 18n25

Post-Colloquial (后口语): 318-320, 334

Post-New-Tide Poetry (后新诗潮): 421

Post-Obscure Poetry (后朦胧诗): 17-18, 65, 408

Pound, Ezra: 189, 403

print culture: 22

prose poetry: 30, 189, 197-199, 277, 467; chapter Six (definition: 230)

Proust, Marcel: 416

publication (definition): 7-8

Pumin 普珢 (1962): 72

pure poetry (纯诗): 196, 362

Pushkin, Aleksandr: 412

Putonghua 普通话 $\rightarrow$ Standard Language

Qi Guo 祁国 (1968): 39

Qi ling hou yi dai 七O后一代 $\rightarrow$ Post-70

Generation
Qiaoqiao 乔乔: 460

Qimeng 《启蒙》 $\rightarrow$ Enlightenment

Qin Bazi 秦巴子 (1960): 105-107, 124

Qingxiang 《倾向》 $\rightarrow$ Tendency

Qu Yuan 屈原: 1, 16, 108, 136-137, 178, 225, 386, 424

quotidianism (日常主义): 252

reading the poet: $2,120-123,173,359$,

$377-378$; $\rightarrow$ text is like the person

recitation: 1, 29, 104 199-200, 233-235, 278-280, 294-301, 315, 329, 394;

chapter Thirteen

Reggio, Godfrey: 466

regional language (方言) $\rightarrow$ dialect

rhythm $\rightarrow$ poetic rhythm

richangzhuyi 日常主义 $\rightarrow$ quotidianism

Rilke, Rainer Maria: 403

Rimbaud, Arthur: 105, 108-109, 204, 220, 362

romanticism: xiii, 37, 195, 203, 208, 212-214, 235, 264, 360-361, 372, $375,380,392,394,411$; passim in chapter Three; $\rightarrow$ Romanticism

Romanticism: xiii, 4, 16, 25n33, 106107, 110, 360; $\rightarrow$ romanticism

Root-Seeking (寻根): 17, 190, 245, 256, 303

Rubbish School (垃圾派) $\rightarrow$ Trash School

Rumsfeld, Donald: 465-467

Rupture (断裂): 389-390

Saddam Hussein: 465-466

Safran, William: 143

Said, Edward: 141, 160

Sajiao shikan《撒娇诗刊》 $\rightarrow$ Coquetry Poetry fournal

Sajiaopai 撒娇派 $\rightarrow$ Coquetry School

Salinger, Jerome David: 6

Same Generation (同代): 68

sanwenshi 散文诗: 225 ; $\rightarrow$ prose poetry

Saussy, Haun: 57, 139

Sax, Broia: 110

Scar Literature (伤痕文学): 320

second channel (第二渠道、二渠道):

9n9

Selected Post-80 Poetry (八○后诗选): $18 \mathrm{n} 25$

Shang Qin 商禽: 229 
Shang Zhongmin 尚仲敏 (1964): 71

Shanghen wenxue 伤痕文学 $\rightarrow$ Scar Literature

Shao Fei 绍飞: 150

Shao Jian 邵建: 435

Shelley, Percy Bysshe: xiii, 107, 169, 228,412

Shen Haobo 沈浩波 (1976): 20, 52, 59, 152-153, 468; chapter Nine; passim in Twelve (esp 405-406); $\rightarrow$ Choushui; $\rightarrow$ Shen Lang

Shen Lang 沈浪 (= Shen Haobo): 415

Shen Qi 沈奇: 72n12, 420, 422-423, 425, 427, 439

Sheng Xing 盛兴 (1978): 335, 436

Shi cankao 《诗参考》 $\rightarrow$ Poetry Reference

Shi Guanghua 石光华 (1958): 17

Shi jianghu 《诗江湖》 $\rightarrow$ Poetry Vagabonds

Shi Jile 释极乐 (= Zhong Ming): 133n62

Shi Mingzheng 施明正: 113

shi sanwen 诗散文: 225 ; $\rightarrow$ poetic prose

Shi wenben 《诗文本》 $\rightarrow$ Poetry Text

shi yan zhi 诗言志 $\rightarrow$ poetry articulates....

Shige yu ren 《诗歌与人》 $\rightarrow$ Poetry and People

Shïing 《诗经》 $\rightarrow$ Book of Songs

Shiren xiezuo 诗人写作 $\rightarrow$ Poets' Writing

shitan 诗坛 $\rightarrow$ poetry scene

shiyan 实验 $\rightarrow$ experimental

shiyi 诗意 $\rightarrow$ poetic sentiment

Shizhi 食指 (= Guo Lusheng, 1948):

15, 101, 151, 249, 438; $\rightarrow$ Guo

Lusheng

Shu 《書》 $\rightarrow$ Writing

Shu 坚 (1972): 335

Shu Ting 舒婷 (1952): 7, 31, 34, 70-71, 91, 93n1, 101, 106, 159n40, 249, 341

shushang 书商 $\rightarrow$ book brokers

Simon, John: 225, 227, 229, 240, 246

sinologists: 54-59, 319, 382, 388-390, $403,430,437,444$

social concern: 20, 305, 326, 340-342

Solzhenitsyn, Aleksandr: 6

Song Lin 宋琳 (1959): 139, 461

Song Qu 宋渠 (1963): 17

Song Wei 宋炜 (1964): 17

Song Xiaoxian 宋晓贤 (1966): 152, 431

Song Zuifa 宋醉发: 38-39, 138

Songs of the South (楚辞): 1, 424
Sontag, Susan: 416

Sötemann, August Lammert: 347, 360-361

soulmate journals (同仁刊物、同人刊 物): 444

South $\rightarrow$ North and South....

Southern Poetry Review (南方诗志): 154, 444-445

Spalek, John: 146, 179

spiritual pollution (精神污染): 16, 35, $147,149,162,423,447-448$

Standard Language (普通话): 266-267, 373-374, 376, 387-388, 407-408, 410

Stein, Gertrude: 220

Su Shaolian 苏绍连: 229

Su Shi 苏轼: 1, 413

Su Wei 苏炜: 63, 71, 77, 163

subjectification: chapter Seven (esp 252-274)

sublime: $25 \mathrm{n} 33$

suicide and literature: chapter Three

Suleiman, Susan: 160

Sun Shaozhen 孙绍振: 417, 421, 423

Sun Wenbo 孙文波 (1956): xiii, 20, 52, 59, 83, 169, 249, 309, 461; chapter Eight; passim in Twelve

Sun Wukong 孙悟空: 404

Sunflower (葵): 331

Survivors, Survivors (幸存者): 95, 119

Tabori, Paul: 143, 159-160

Tagore, Rabindranath: 412

Taiwan poetry: 53

Tamen 《他们》 $\rightarrow$ Them

Tan Chee-Lay: 162, 172

Tan Wuchang 谭五昌: 102, 124

Tang Jin 唐晋: 421

Tang Xiaobing: 25

Tang Xiaodu 唐晓渡: xiv, 130, 152, $161,184,281,393,461$; passim in chapter Twelve

Tang Xin 唐欣: 152

tansuo 探索 $\rightarrow$ explorative

Tao Naikan 陶乃㑆: 386-389

Teeuwen, Rudolphus: 178

Tendency (倾向): 141 (edited by Beiling); 17, 100, 135, 189-190, 402, 444-445 (edited by Chen Dongdong et al) text $\rightarrow$ text, context and metatext text, context and metatext (definition): $1,13,50$ 
text is like the person (文如其人): 192， 446 ; $\rightarrow$ reading the poet

thanatography: 438; chapter Three (definition: 93 )

Them (他们): 16, 63, 72-76, 78, 193, 250, 252, 261, 338, 341, 365, 380, $396,409,438,445$

theory and methodology: 59-60

Third Generation (第三代): 17-18, 65, 77, 334, 408, 427-428, 444

Thug Literature (流讯文学、㾂子文 学) $\rightarrow$ Hooligan Literature

Tian Jian 田间: 4, 102

Tian Yong 田涌: 419

Tiantian 田田: 150

ties of allegiance (关系): 57, 399, 441-450

Today (今天): 7, 15-16, 20, 26n 35,42 , 65-76, 101, 113, 141, 147, 149, 151, 153, 160, 168n53, 185, 254, 370, 380, 409, 437, 444, 447

Tong Wei 童蔚 (1956): 461

Tongdai 《同代》 $\rightarrow$ Same Generation tongren kanzeu 同仁刊物、同人刊物 $\rightarrow$ soulmate journals

traditional Chinese poetics: 2, 15, 30, $33,48,54,107,114-116,121,123$, 146, 173, 185, 192, 228, 253, 261, 287, 302-303, 314, 321, 341-342, $352,354,357,361-362,377-378$, 382-383, 396, 403, 447-448

Transitive Writing (及物性的写作): 303

Trash School (垃圾派): 20, 338

Tsvetayeva, Marina: 6, 108

Twitchell-Waas, Jeffrey: 65, 71

underground (地下): 6-7, 12, 15, 30, 32， 38-39, 141, 145, 151

unofficial (非官方): chapter One (esp 5-12) et passim

Van Ark, Daniëlle: 473

Van Gogh, Vincent: 105, 108, 260

Vandermeer, Pieter: 64, 140, 142, 248

verse-external poetics: 263, 267, 278, 303, 331, esp 345-346 (definition: 345), 348, 399

Vision (目光): 22, 464-465

Visser, Robin: 321

visualization: $38-39,45-47,335$

voice $\rightarrow$ poetic voice
Vroegindeweij, Victor: 473

vulgarization: 18, 52, 135, 189, 214 , 362,445

Wah, Fred: 357

Wan Xia 万夏 (1962): 17, 39, 43, 97n7, 116,259

Wan Zhi 万之: 163

Wang Ai 王艾 (1971): 334

Wang Ban: 25, 125

Wang Bin 王涁: 150

Wang, David Der-wei: 53, 113

Wang Guangming 王光明: 225-226

Wang Guozhen 汪国真: 24, 106, 408

Wang Jiaxin 王家新 (1957): 19, 52, 83, 103, 106-108, 119, 249, 281, 309, 315, 341, 387, 393; chapter Four; passim in Twelve (esp 432-434); $\rightarrow$ $\mathrm{Zi} \mathrm{An}$

Wang Jing: 13, 321

Wang Shuo 王朔: 247, 321

Wang Wei 王巍: 421, 423

Wang Xiaobo 王晓波: 438

Wang Xiaoni 王小妮 (1955): 406

Wang Yichuan 王一川: 70, 125, 251

Wang Yin 王寅 (1962): 72

Wang Yuechuan: 111-112

Wang Zheng 汪政: 251

Wang Zhong 王中

Wangluo wenxue 网络文学 $\rightarrow$ Web Literature

Web Literature (网络文学): 330

Wedell-Wedellsborg, Anne: 178

Wei Tianwu 魏天无: 281, 303, 450

Wei'an 苇岸: 119

Weihui 卫慧: 321-322

Wen Jie 闻捷: 113

wen ru qi ren 文如其人 $\rightarrow$ text is like the person

Wen Tingyun 温庭云: 403

wen yi zai dao 文以载道 $\rightarrow$ literature to convey the Way

Wen Yiduo 闻一多: 4, 147, 286, 299

Wen Yuanhui 温远辉: 191

wenhua jüngi 文化经济 $\rightarrow$ cultural economy

wenhua re 文化热 $\rightarrow$ high culture fever

Wenmang 文盲 (1977): 341, 468

wenren xiang qing 文人相轻.... $\rightarrow$ literary

men disparage one another....

Wenxue geming 文学革命 $\rightarrow$ Literary

Revolution 
"West," modern Chinese poetry's relation to and image of: 15,25 , 54-59, 107, 145, 266-267, 317, 339, 378-379, 382-390; chapter Twelve

Whitman, Walt: 125, 249, 387, 412

Wholism (整体主义): 17

Wiedenhof, Jeroen: colophon

Wigman, Menno: 226

Wilde, Oscar: 38

Wings (翼): 461

Women's Poetry (女性诗歌), women's writing and feminist critical discourse: 17, 46, 53, 102, 309, 321, 335, 340, 347, 355, 389, 399, 414, 430, 432, 461

women's writing $\rightarrow$ Women's Poetry....

Wong, Lawrence Wang-chi: 147

Wordsworth, William: 228

Writers' Association $\rightarrow$ Chinese Writers' Association

Writing (書): 462

Wu Ang 巫昂 (1974): 335

Wu Kaijin 吴开晋: 66, 72n12

Wu Quan 武权: 23, 462-463, 465, 472

Wu Shaoqiu 吴少秋: 133n62

Wu si 五四 $\rightarrow$ May Fourth

Wu Sijing 吴思敬: 33, 192, 203, 335, 421, 423, 425

Wu Wenguang 吴文光: 259-263, 330

Wu Xiaodong 吴晓东: 102, 110

Wu Xinhua 吴新化: 33

Xi Chuan 西川 (1963): xiv, 11, 13, 17, 26-30, 37, 47-48, 52-53, 59, 83 , 95-123, 125, 127n51, 135, 168, 249, 274, 298, 309, 315, 342, 365-366, 392, 397, 467; chapters Five, Six and Ten; passim in Twelve; $\rightarrow$ Liu Jun

Xi Du 西渡 (1967): 399, 406, 417 , 419-420, 425-426, 434-435

Xi Mi 奚密 $\rightarrow$ Yeh, Michelle

Xi Yunshu 席云舒: 332, 334

Xia Yuanming 夏元明: 251

Xiabanshen 《下半身》 $\rightarrow$ Lower Body

Xiandai Hanshi 《现代汉诗》 $\rightarrow$ Modern Han Poetry

Xiandaishi neibu jäaliu ziliao 《现代诗 内部交流资料》 $\rightarrow$ Modern Poetry Materials for Internal Exchange xianfeng 先锋 $\rightarrow$ avant-garde

Xiang Weiguo 向卫国: 332
Xiangwang 《象罔》 $\rightarrow$ Image Puzzle

Xiangzi 湘子 (= Yu Jian?): 429

Xiao An 小安 (1964): 436-437

Xiao Hai 小海 (1965): 72, 72n12

Xiao Jun 小君 (1962): 72

Xiao Kaiyu 肖开愚 (1960): 20, 83, 139, $169,249,281,283,303,341,402-$ 403, 406, 430, 442

Xiao Quan 肖全: 39

Xiao Ying 肖鹰: 101, 111

Xiaohua 晓华: 251

Xiaoxiao (China, ㅇ) 潇潇 (1962): 461

Xiaoxiao (Taiwan, ठ) 萧萧: 225-227

Xiaoyuan shige 校园诗歌 $\rightarrow$ Campus Poetry

Xie Linglan 谢凌岗: 102, 110

Xie Mian 谢冕: 32-33, 102, 130, 250, 421

Xie Ye 谢烨: 31, 91, 391, 414

Xie Youshun 谢有顺: 251, 261, 333, 388, 393, 396; passim in chapter Twelve (esp 415-417)

Xin qingnian 《新青年》 $\rightarrow$ New Youth

Xin shichao 新诗潮 $\rightarrow$ New Tide Poetry

$X i n$ shidai 新时代 $\rightarrow$ New Period

Xin wenhua yundong 新文化运动 $\rightarrow \mathrm{New}$ Culture Movement

Xin wenxue 新文学 $\rightarrow$ New Literature

Xinsh $i$ 新诗 $\rightarrow$ New Poetry

Xin Yue 辛月: 251

Xingcunzhe 幸存者 $\rightarrow$ Survivors

Xinshengdai 新生代 $\rightarrow$ Newborn Generation

Xu Jiang 徐江 (1967): 105-107, 152, 314, 318, 331, 333; passim in chapter Twelve (esp 412-415)

Xu Jingya 徐敬亚 (1949): 18, 20, 73, 190, 347

Xu Yi 胥式: 104

Xu Zhimo 徐志摩: 4, 106, 287

Xuanyuanshike 轩辕轼轲 (1971): 335

Xue Di 雪迪 (1957): 139, 230

Xungen 寻根 $\rightarrow$ Root-Seeking

Xushish $i$ 叙事诗 $\rightarrow$ Narrative Poetry...

Yan Jun 颜峻 (1973): xiii, 22, 27, 29-30, 47, 53, 341-343; chapter Thirteen

Yan Li 严力 (1954): 139, 141, 431

Yang Changzheng 杨长征: 192, 194, 197, 203 
Yang Ke 杨克 (1957): 39, 191, 332; passim in chapter Twelve

Yang Lan: $5 \mathrm{n} 4$

Yang Li 杨黎 (1962): 17, 38, 259, 339340, 391, 408

Yang Lian 杨炼 (1955): xv, 7, 18, 34, 52, 68-69, 93n2, 135, 230, 247, 365, 408; chapter Four; $\rightarrow$ Fei Sha

Yang Lihua 杨立华: 172

Yang Siping 杨四平: 172

Yang Xiaobin 杨小斌: 161, 164, 172, 176, 440, 461

Yeats, William Butler: 403-405

Yeh, Michelle: 3, 18, 25, 33, 49, 53, 55, 63, 91, 102-103, 107-108, 112-113, $117,130,172,190,251,349,362$, 366, 372, 446, 448, 450

Yemen 也门 (= Yi Sha): 153n30

Yi 《翼》 $\rightarrow$ Wings

Yi hang 《—行》 $\rightarrow$ First Line

Yi Sha 伊沙 (1966): 20, 24, 38-39, 41, 44, 48, 105-107, 152-153, 309, 314, 318, 319n14, 333, 338-340; passim in chapter Twelve; $\rightarrow$ Yemen

Yin Lichuan 尹丽川 (1973): 20, 27, 29-30, 47, 52, 461, 468; chapter Nine

Yiping 一平: $161,172,180$

Yo Yo 友友: 147, 153

Yu Guangzhong 余光中: 106

Yu Hong 余虹: 102, 110

Yu Hua 余华: 247, 468

Yu Jian 于坚 (1954), 11, 26-30, 37-38, 47, 52, 71-72, 78, 83-84, 86, 107, 132-133, 187, 283, 309-310, 315, 318, 325, 338-339, 346-347, 361, 363; chapters Six, Seven and Eleven; passim in Twelve (esp 407-412); $\rightarrow$ Dawei; $\rightarrow$ Xiangzi

Yu Liwen 余丽文: 251

Yu Luoke 遇罗克: 69

Yu Xiaowei 于小韦 (1961): 72

Yu Xugang 余徐刚: 102, 115-116

Yuanchuangxing xiezuo 《原创性写作》 $\rightarrow$ Original Writing

Yufeng 宇峰: 153

Zang Di 㶓棣 (1964): 83-84, 167, 402, 406, 418n10, 419-420, 425-426, 432, 461

Zang Kejia 縅克家: 4

Zang Li 藏力 $\rightarrow$ Zang Di

Zeng Hong: 112
Zha Haisheng 查海生 (= Haizi): xiv, 96-97

Zhai Yongming 翟永明 (1955): 17, 46, 53, 83, 139, 402-403, 408, 432

Zhang Chengzhi 张承志: 113

Zhang Hong 张闳: 33, 172, 176, 180

Zhang Hongming 章宏明: 427-428

Zhang, Jeanne Hong: 53

Zhang Longxi: 2, 55, 354, 357

Zhang Ning 张柠: 251

Zhang Qinghua 张清华: 102, 333, 419, $421 ; \rightarrow$ Jing Wen

Zhang Shuguang 张曙光 (1956): 20, 83, 169, 281, 303, 402-404, 406, 416, 420, 431, 436, 443

Zhang Wei 张维: 100

Zhang Xiafang 张夏放: 83, 85, 87

Zhang Xudong: 57, 139

Zhang Yingjin: 55

Zhang Zao 张來 (1962): 70n9, 83, 139, 161,172

Zhang Zhen 张真 (1962): 139, 144, 185

Zhang-Kubin, Suizi: 151

Zhao, Henry: 34-36, 45, 112n33, 143n5

Zhao Qiguang: 112

Zhao Xun 赵㻏: 168

Zhao Yiheng 赵毅衡 $\rightarrow$ Zhao, Henry

Zhao Zhenkai 赵振开 (= Bei Dao): xiv zhebi 遮蔽 $\rightarrow$ obstruction

Zheng Min 郑敏: 4, 423

Zheng Yi 郑义: 163

Zhengtizhuyi 整体主义 $\rightarrow$ Wholism

Zhengzhi shuqingshi 政治抒情诗 $\rightarrow$

Political Lyricism

Zhishi qingnian 知识青年 $\rightarrow$ Intellectual Youths

Zhishifenzi xiezuo 知识分子写作 $\rightarrow$

Intellectual Writing

Zhong Ming 钟鸣 (1953): 38, 102, 118n40, 133n62, 444; $\rightarrow$ Shi Jile

Zhongdao 中岛 (1963): xiv, 399, 431

Zhongguo dangdai shiyan shige 《中国当代 实验诗歌》 $\rightarrow$ Contemporary Chinese Experimental Poetry

Zhongguo zuojia xiehui 中国作家协会 $\rightarrow$

Chinese Writers' Association

Zhongjiandai 中间代 $\rightarrow$ Middle

Generation

Zhongnian xiezuo 中年写作 $\rightarrow$ Middle-

Aged Writing

Zhou Jun 周俊: 100 
Zhou Lunyou 周伦佑 (1952): 17, 26

Zhou Weihui 周卫慧 $\rightarrow$ Weihui

Zhou Yubing 周玉冰: 102, 115-116

Zhou Zan 周瓒 (1968): 334, 440, 461

Zhu Dake 朱大可: 101-102, 110,334, 339

Zhu Jian 朱剑 (1976): 335

Zhu Wen 朱文 (1967): 368, 370, 373, 375, 379, 382-384, 389, 391, 396, 408
Zhu Xiaoyang 朱小羊: 260, 262

Zhuang Rouyu 庄柔玉: 180

Zhuangzi 庄子: 219, 382

Zi An 子岸 (= Wang Jiaxin): 168n53, 440

Zou Jianjun 邹建军: 132

Zou Jingzhi 邹静之 (1952): 102

Zuozhuan 《左传》: 359 